\title{
Failure maps and optimal design of metallic sandwich panels with truss cores subjected to thermal loading
}

\author{
Wu Yuan, Hongwei Song*, Chenguang Huang \\ Key Laboratory for Mechanics in Fluid Solid Coupling Systems, Institute of Mechanics, Chinese Academy of Sciences, No. 15 Beisihuanxi Road, Beijing 100190, \\ China
}

\section{A R T I C L E I N F O}

\section{Article history:}

Received 24 February 2016

Received in revised form

31 May 2016

Accepted 6 June 2016

Available online 7 June 2016

Keywords:

Optimization

Truss core

Sandwich panel

Failure mode

\begin{abstract}
A B S T R A C T
Sandwich panels with truss cores have been widely investigated due to their superior mechanical performances. When being used in the thermal protection system of a high-speed aircraft, sandwich panels are usually subjected to intense thermal loading and may fail due to various mechanisms. This paper presents a theoretical and numerical analysis on the failure mechanisms and optimal design of metallic sandwich panels with truss cores subjected to uniform thermal loading. Five failure modes are considered: global buckling, face sheet buckling, face sheet yielding, core member buckling and core member yielding. Failure maps of sandwich panels with several truss core topologies are developed based on these failure modes. Taking the five failure modes as constraint conditions, sandwich panels with truss cores are optimally designed for the minimum weight at given thermal loadings. It is found from the optimal analysis that sandwich panels with Kagome and X-type truss cores are more efficient than those with tetrahedral and pyramidal truss cores. Sandwich panels with fully-clamped boundary conditions have superior thermal loading resistance than those with simply-supported boundary conditions.
\end{abstract}

(c) 2016 Elsevier Ltd. All rights reserved.

\section{Introduction}

Sandwich panels with truss cores (SPTCs) are a class of novel structures that can be applied as both load bearing components and other functionality, such as thermal management, energy absorption and blast resistance [1-5]. A prominent characteristic of SPTCs is that their macroscopic mechanical behavior can be designed or tailored through the configuration, arrangement and material of mesostructure, of the lattice truss. There have been a variety of configurations of lattice truss materials, such as pyramidal [6,7], tetrahedral [8-11], Kagome [12,13] and recently proposed X-type [14-17]. Compared with closed or open foams, which are bending-dominated configuration, the stretchingdominated lattice truss material that have high degree of nodal connectivity is much stiffer and stronger [18]. When being used as thermal protection systems of high speed vehicles, sandwich panels typically experience a large temperature change. The hightemperature degraded properties together with intense thermal loading may lead to a failure of various mechanisms. Therefore, the failure behavior of sandwich panels to thermal loading becomes a driving design parameter before they can be applied into practice.

\footnotetext{
* Corresponding author.

E-mail address: songhw@imech.ac.cn (H. Song).
}

There have been some studies on the structural response of SPTCs at room temperature. In some cases, SPTCs have been tested in various shear and bending modes $[8,9,19]$. To study the in-plane compressive behaviors, Cote et al. [7] carried out experimental and theoretical analysis on the response of metallic sandwich columns with pyramidal truss cores made from AISI 304 stainless steel. Failure maps of the sandwich column are constructed based on three failure mechanisms: Euler buckling, shear buckling and face sheet wrinkling. For all-composite sandwich columns, face sheet crushing will appear besides the three failure modes considered in metallic sandwich columns [20]. Wicks and Hutchinson [21] carried out theoretical analysis on the optimal design of sandwich panels with either planar trusses or solid face sheets with a single material subjected to prescribed combinations of bending and transverse shear loading. Four failure modes are considered in their analysis: face yielding, face buckling, core member yielding and core member buckling. Based on these failure modes, Zok et al. [22] obtained the failure mechanism map of sandwich beams with pyramidal truss cores and compared with the three point bending test. Then, Rathbun et al. [23] conducted an systematic optimal analysis on sandwich beams with several core topologies, including pyramidal and tetrahedral truss cores, square honeycombs, and corrugated sheets. In their works, the optimal design is obtained at the confluence of three failure mechanisms. However, it should be noted that for a nonlinear system, the global optimal 
result is not necessarily in the intersection of constraint equations, which have obvious nonlinear characteristics in nature. In this case, the numerical programming of the optimization model is imperative.

When structures are subjected to high temperature environments, one of the undesirable effect is the development of thermal stresses, which is often happened at temperatures below those that impair the material properties considerably [24]. Compressive thermal stresses arise either from non-uniform temperature distributions or from supports which constrain the thermal expansion even when heating is uniform. The behavior of global buckling induced by the compressive thermal stresses is an important failure mode for the slender or thin-walled structure, and has been studied extensively for shells and general sandwich plates in the theoretical analysis [25-30]. Rakow and Waas [31] also carried out experimental analysis on the thermal buckling behavior of sandwich panels with foam cores. For SPTCs, Yuan et al. [32] obtained the eigenvalue buckling and post buckling behavior of fullyclamped (CCCC) and simply-supported (SSSS) SPTCs subjected to uniform thermal loading. Later on, Yuan et al. [33] also performed experimental study on the thermal buckling behavior of SPTCs, and the full field buckling history of the panel under uniform high temperature environments was obtained. It is found that, due to defects during fabrication, the sandwich panel deformed in asymmetric mode in high temperature environments. However, it should be noted that due to the complexity of the structure, both the face sheet and the core member of the SPTC may fail in various modes, besides global buckling.

Within the authors' knowledge, there has been little theoretical analysis reported on the failure behaviour of SPTCs subjected to uniform thermal loading. In the present paper, five failure mechanisms are considered to obtain the high temperature failure maps of SPTCs, they are global buckling (GB), face sheet buckling (FB), face sheet yielding (FY), core member buckling (CB) and core member yielding $(\mathrm{CY})$. The objective of this paper is to construct failure mechanism maps as well as to estimate the minimum weight design of SPTCs at a given thermal loading with the competing failure modes. The outline of the paper is as follows. Firstly, analytical expressions for critical loads of five failure modes are derived for the CCCC and SSSS SPTCs made from a single metallic material. Based on these expressions, failure mechanism maps are constructed with dimensionless geometrical parameters of SPTCs. Finally, minimum weight designs are obtained for sandwich panels with different truss core topologies by using the numerical optimal program model based on Lingo. It is verified that for this nonlinear problem, the confluence of constraint equations for various failure modes is not the optimal design.

In the present paper, failure maps of SPTCs subjected to uniform thermal loading are developed by comparing the load capacity in these mechanisms. In addition, optimal designs of the SPTC are obtained by using the failure modes of SPTCs as constraint conditions, and the dimensionless weight as objective function. Therefore, analytical expressions of SPTCs under the five failure modes should be deduced.

\section{Failure modes of metallic SPTCs}

Before proceeding, performance evaluation criteria for SPTCs are needed. In an optimization process, one needs to ascertain the minimum weight of SPTCs that can maintain structural integrity at a given thermal loading. Therefore, two dimensionless parameters, one is based on weight and the other based on load, are considered. The pertinent load index for strength-based designs can be expressed as

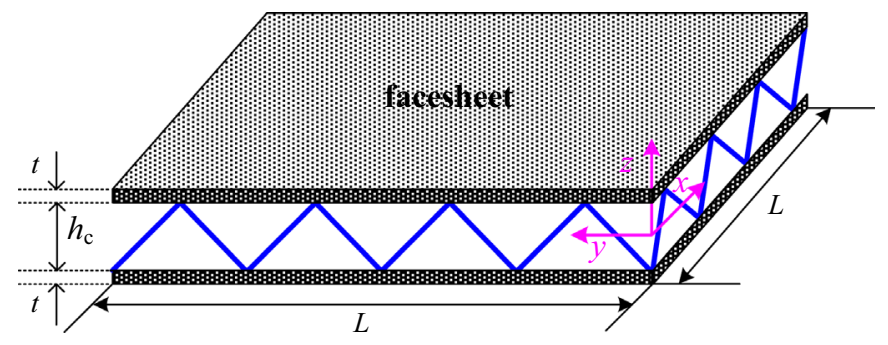

Fig. 1. Schematic of sandwich panels with truss cores.

$\prod_{t}=a \Delta T$

where $\alpha$ and $\Delta T$ are the coefficient of thermal expansion of the material of the SPTC and the temperature rise respectively. The dimensionless weight per unit area of the sandwich panel is [21]

$\Psi=\frac{W}{\rho L}$

where $W$ is the structural weight per unit area and $\rho$ is the density of the solid material.

As illustrated in Fig. 1, consider a square SPTC of length $L$ subjected to uniform thermal loading. The sandwich panel is composed of solid face sheets and truss cores, and the cross section of core member is square. Both face sheets and truss cores are made from the same metallic material. Truss core configurations of SPTCs studied in the present paper, as Table 1 shows, are pyramidal, tetrahedral, X-type and Kagome, respectively. In general, the SPTC is characterized by five independent parameters: face sheet thickness $t$, cores thickness $h_{\mathrm{c}}$, core member thickness $t_{\mathrm{c}}$, the angle between the core member and the face sheet $\theta$, and the length of the square sandwich panel $L$. For the pyramidal, X-type, tetrahedral and Kagome configuration, $\theta$ are $45^{\circ}, 45^{\circ}, 55.7^{\circ}$ and $55.7^{\circ}$ respectively. The core member length $l_{\mathrm{c}}$ is correlated to core thickness $h_{\mathrm{c}}$ by

$l_{c}=\frac{h_{c}}{\sin \theta}$

\subsection{Global buckling (GB)}

For the SPTC which has a thin thickness, global buckling is the main failure mode. Two kinds of boundary conditions are considered: SSSS and CCCC. By ignoring the flexural rigidity of the core, and considering the shear stiffness of the sandwich panel is only contributed by truss cores, equilibrium equations of the sandwich panel with truss cores subjected to uniform thermal loading can be expressed as [32]

$D\left(\frac{\partial^{2} \phi_{x}}{\partial x^{2}}+\frac{1-\mu}{2} \frac{\partial^{2} \phi_{x}}{\partial y^{2}}+\frac{1+\mu}{2} \frac{\partial^{2} \phi_{y}}{\partial x \partial y}\right)+C\left(\frac{\partial w}{\partial x}-\phi_{x}\right)=0$

$D\left(\frac{\partial^{2} \phi_{y}}{\partial y^{2}}+\frac{1-\mu}{2} \frac{\partial^{2} \phi_{y}}{\partial x^{2}}+\frac{1+\mu}{2} \frac{\partial^{2} \phi_{x}}{\partial x \partial y}\right)+C\left(\frac{\partial w}{\partial y}-\phi_{y}\right)=0$

$D\left(\frac{\partial^{2} w}{\partial x^{2}}+\frac{\partial^{2} w}{\partial y^{2}}-\frac{\partial \phi_{x}}{\partial x}-\frac{\partial \phi_{y}}{\partial y}\right)+N \nabla^{2} w=0$

where $u, v$ and $w$ are displacements in $x, y$, and $z$ directions, while $\phi_{x}$ and $\phi_{y}$ are rotations of the normal in the $x z$ and $y z$ planes, 
Table 1

Schematics of SPTCs with the four types of truss core configurations.

\begin{tabular}{ll}
\hline SPTC & truss core \\
\hline
\end{tabular}

pyramidal configuration
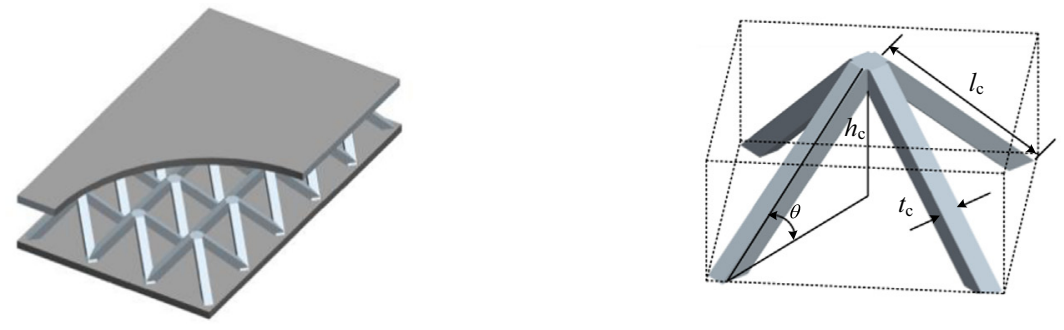

tetrahedral configuration
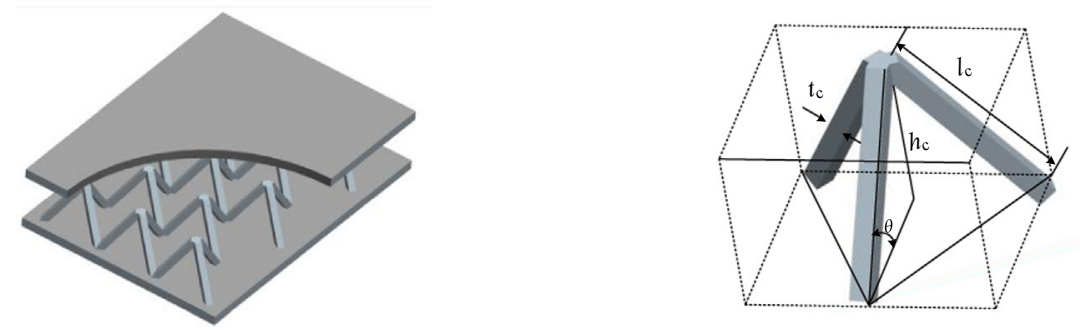

X-type configuration
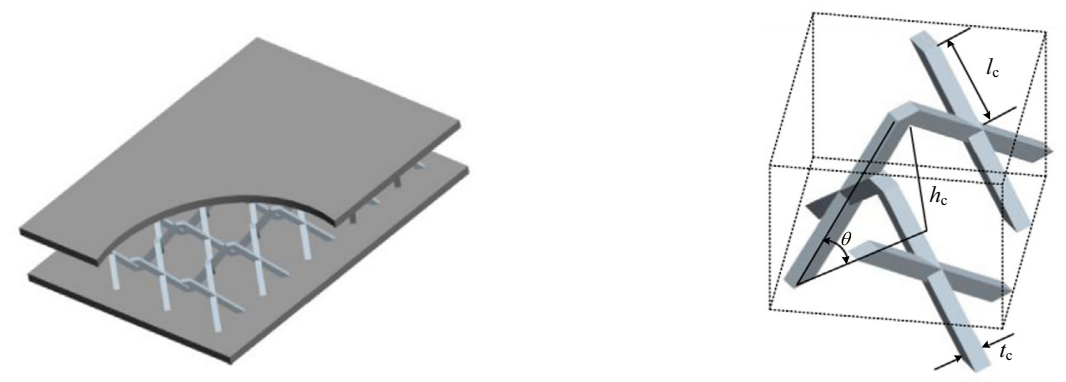

\section{Kagome configuration}
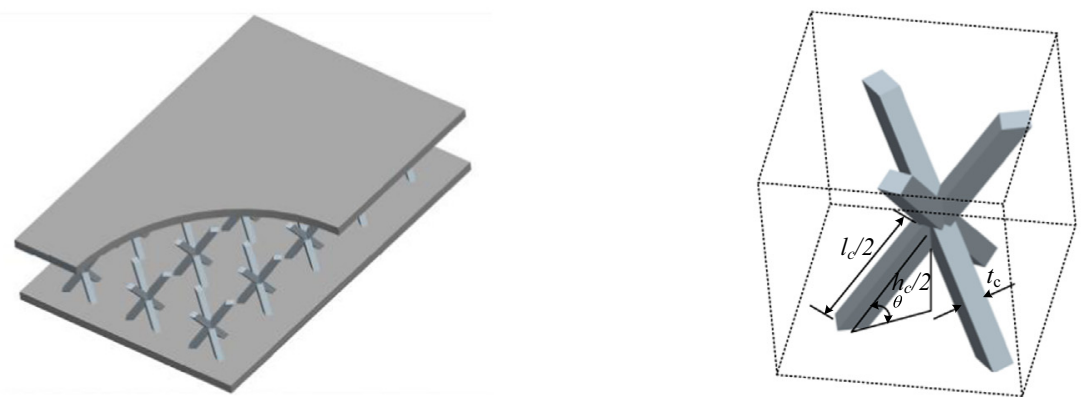


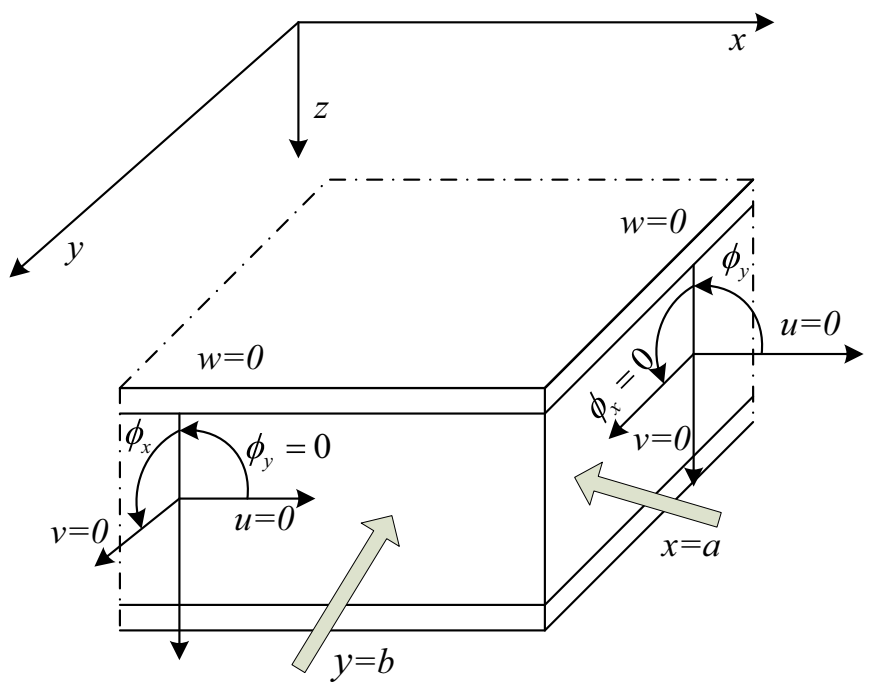

Fig. 2. Boundary conditions of SSSS SPTCS when $x=a$ and $y=b$.

respectively. $N$ is the in-plane compressive force. $D$ is the flexural stiffness of the SPTC, which can be computed as

$D=\frac{E_{c}\left(h_{c}+t_{f}\right)^{2} t_{f}}{2\left(1-\mu^{2}\right)}$

where $E_{c}$ and $\mu$ are the elastic modulus and poisson's ratio of the solid material. And the shear stiffness of the four configurations of SPTCs illustrated in Table 1 is given by

$C_{\text {pyramid }}=C_{X-\text { type }}=\frac{E_{c} A_{c} \sin ^{3} \theta}{h_{c}}$

$C_{\text {tet }}=C_{\text {Kagome }}=\frac{E_{c} A_{c} \sin ^{3} \theta}{\sqrt{3} h_{c}}$

where $A_{c}$ is the cross-sectional area of the core member.

\subsubsection{GB load for SSSS condition}

As shown in Fig. 2, The SSSS condition is given by

$x=0, a: w=\phi_{x}=0$

$y=0, b: w=\phi_{y}=0$

and the following virtual displacement modes are assumed

$\phi_{x}=u_{0} \cos \alpha_{k} x \sin \beta_{l} y$

$\phi_{y}=v_{0} \cos \beta_{l} y \sin \alpha_{k} x$

$w=w_{0} \sin \alpha_{k} x \sin \beta_{l} y$

where $u_{0}, v_{0}$ and $w_{0}$ are Fourier constant coefficients, $\alpha_{k}$ and $\beta_{l}$ are $\frac{k \pi}{a}$ and $\frac{l \pi}{b}$ respectively. Substituting Eqs. (8a-8c) into equilibrium Eqs. $(4 a-4 c)$, the critical buckling temperature of SPTCs can be obtained

$T_{c r}=\frac{(1-\mu)}{E \alpha t} \frac{2 D C \pi^{2}}{C L^{2}+2 D \pi^{2}}$

\subsubsection{GB load for CCCC condition}

For SPTCs under CCCC conditions, the critical buckling temperature of global buckling cannot be analytically solved as those under SSSS conditions, since governing equations for the deformation mode are complicated due to complex boundary conditions. Therefore, displacement solution functions were assumed in the form of two sets of double Fourier series expansions, which can be expressed as

$w=\sum_{m=1}^{\infty} \sum_{n=1}^{\infty} A_{m n} \sin \alpha_{m} x \sin \beta_{n} y$

$\phi_{x}=\sum_{m=1}^{\infty} \sum_{n=1}^{\infty} B_{m n} \sin \alpha_{m} x \sin \beta_{n} y$

$\phi_{y}=\sum_{m=1}^{\infty} \sum_{n=1}^{\infty} C_{m n} \sin \alpha_{m} x \sin \beta_{n} y$

where $A_{m n}, B_{m n}$ and $C_{m n}$ are Fourier constant coefficients, and $\alpha_{m}$ and $\beta_{n}$ are defined as $\frac{m \pi}{a}$ and $\frac{n \pi}{b}$ respectively. The characteristic equations of the SPTC can be obtained by eliminating the unknown variables [32].

To obtain the critical buckling temperature of the SPTC under CCCC condition, a computer program with Fortran code is developed by calling the subroutine GVCRG of IMSL, which is used to solve Eigen value problems.

\subsection{Face sheet buckling (FB)}

When the face sheet is relatively thin and the overall sandwich panel is relatively thick, the face sheet will buckle under compressive thermal stresses. As shown in Fig. 3, the face sheet is assumed to buckle with the node lines illustrated in the figure. The effect of rotation restraint of core members on the face sheet at the point of attachment is neglected to obtain the critical buckling temperature, and this will underestimate somewhat the buckling load. According to geometric configurations of truss cores, the critical load of FB mode can be obtained

$\Delta T_{\text {pyramid }}^{F B}=\frac{\pi^{2}}{12(1+\mu) \alpha}\left(\frac{t}{h_{c}}\right)^{2}$

$\Delta T_{X-t y p e}^{F B}=\frac{5 \pi^{2}}{24(1+\mu) \alpha}\left(\frac{t}{h_{c}}\right)^{2}$

$\Delta T_{\text {tet }}^{F B}=\frac{7 \pi^{2}}{54(1+\mu) \alpha}\left(\frac{t}{h_{c}}\right)^{2}$

$\Delta T_{\text {Kagome }}^{F B}=\frac{14 \pi^{2}}{27(1+\mu) \alpha}\left(\frac{t}{h_{c}}\right)^{2}$

where the subscript denotes the type of truss core configuration.

\subsection{Face sheet yielding $(F Y)$}

For SPTCs with thick face sheets, FY will be the main failure mode. The temperature rise leading to the FY can be computed as 


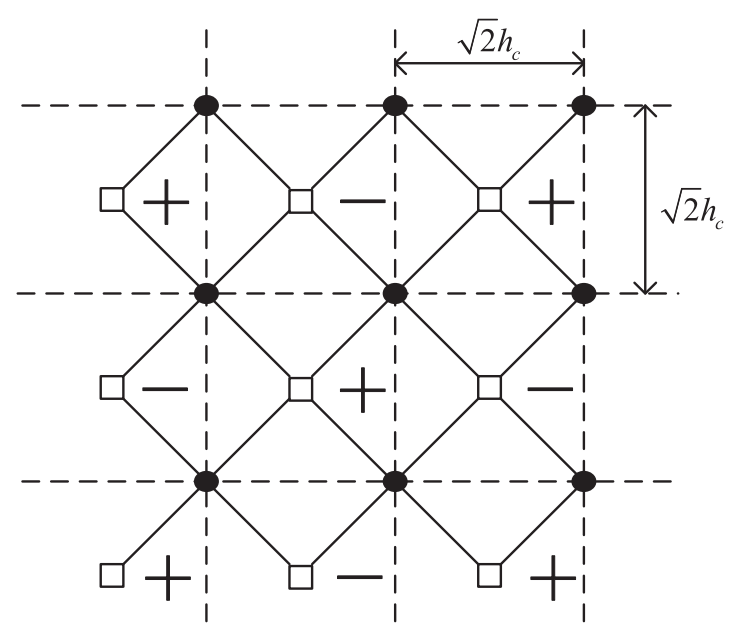

(a)

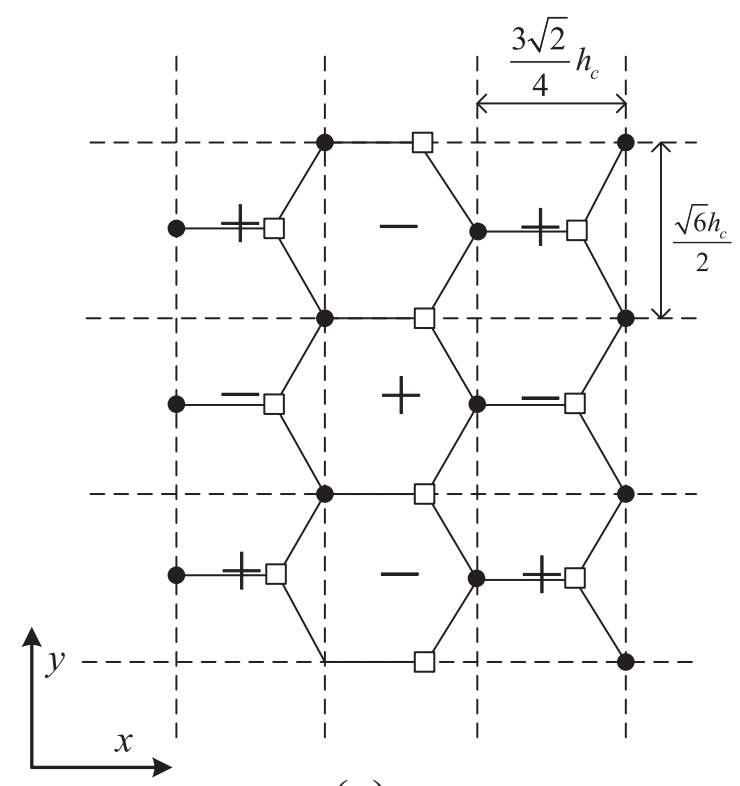

(c)

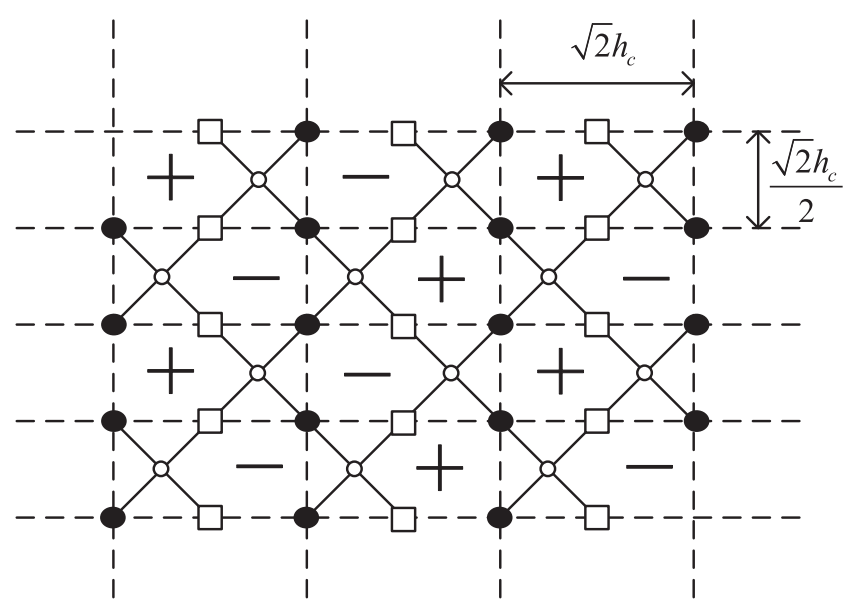

(b)

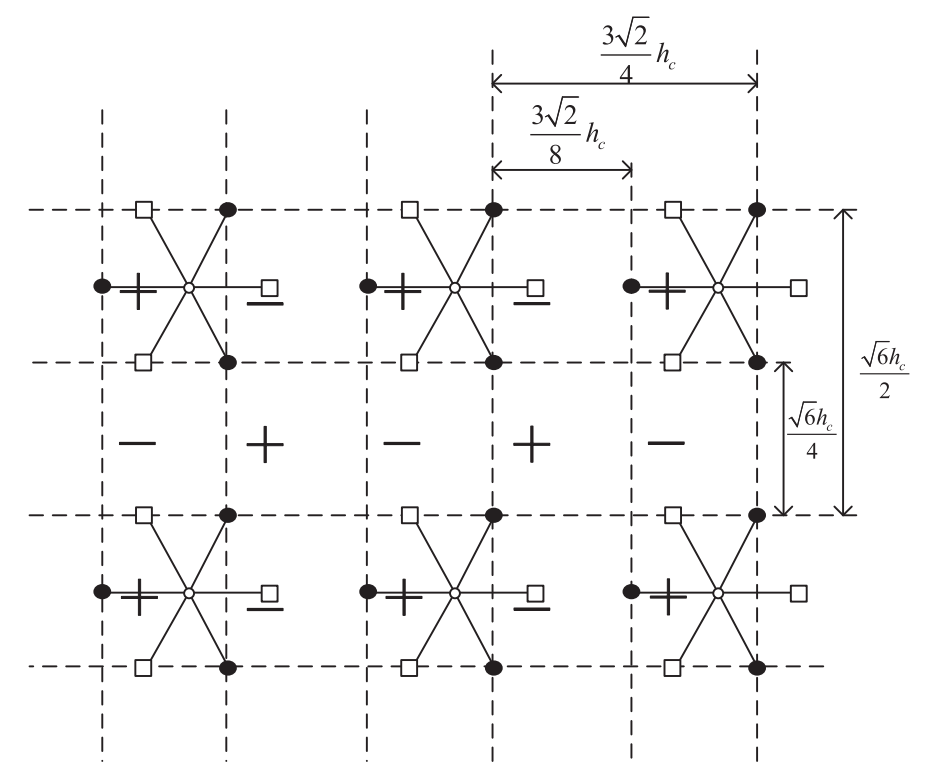

(d)

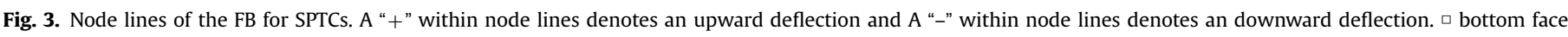

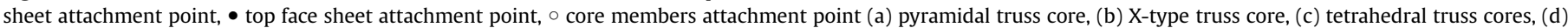
Kagome truss core.

$\Delta T=\frac{\epsilon_{y}}{\alpha}$

where $\varepsilon_{y}$ is the yield strain of the sandwich panel.

\subsection{Core member buckling $(C B)$}

For the sandwich panel with SSSS and CCCC boundary conditions subjected to uniform thermal loading, the deformation of the core member along the out-of-plane direction of the panel is free, nevertheless, the in-plane deformation is constrained. Therefore, the deformation of the core member can be derived by the geometric deformation relation. As shown in Fig. 4, the deformation of the core member produced by the mechanical stress can be expressed as

$\Delta l=\alpha \Delta T l_{c}-\alpha \Delta T h_{c} \sin \theta$
To obtain of the critical load, truss joints are idealized as pin joints offering no rotational resistance between the core member and the face sheet. This assumption is widely used in the SPTC analysis $[21,22]$. However, the assumption underestimates the buckling resistance of the core member, and overestimates the weight of SPTC. The critical buckling temperature of the core member with different configurations can be expressed as

$\Delta T_{\text {pyramid }}^{\mathrm{CB}}=\frac{\pi^{2} t_{c}^{2}}{24 h_{c}^{2} \alpha\left(1-\sin ^{2} \theta\right)}$

$\Delta T_{X-\text { type }}^{C B}=\frac{\pi^{2} t_{c}^{2}}{6 h_{c}^{2} \alpha\left(1-\sin ^{2} \theta\right)}$ 

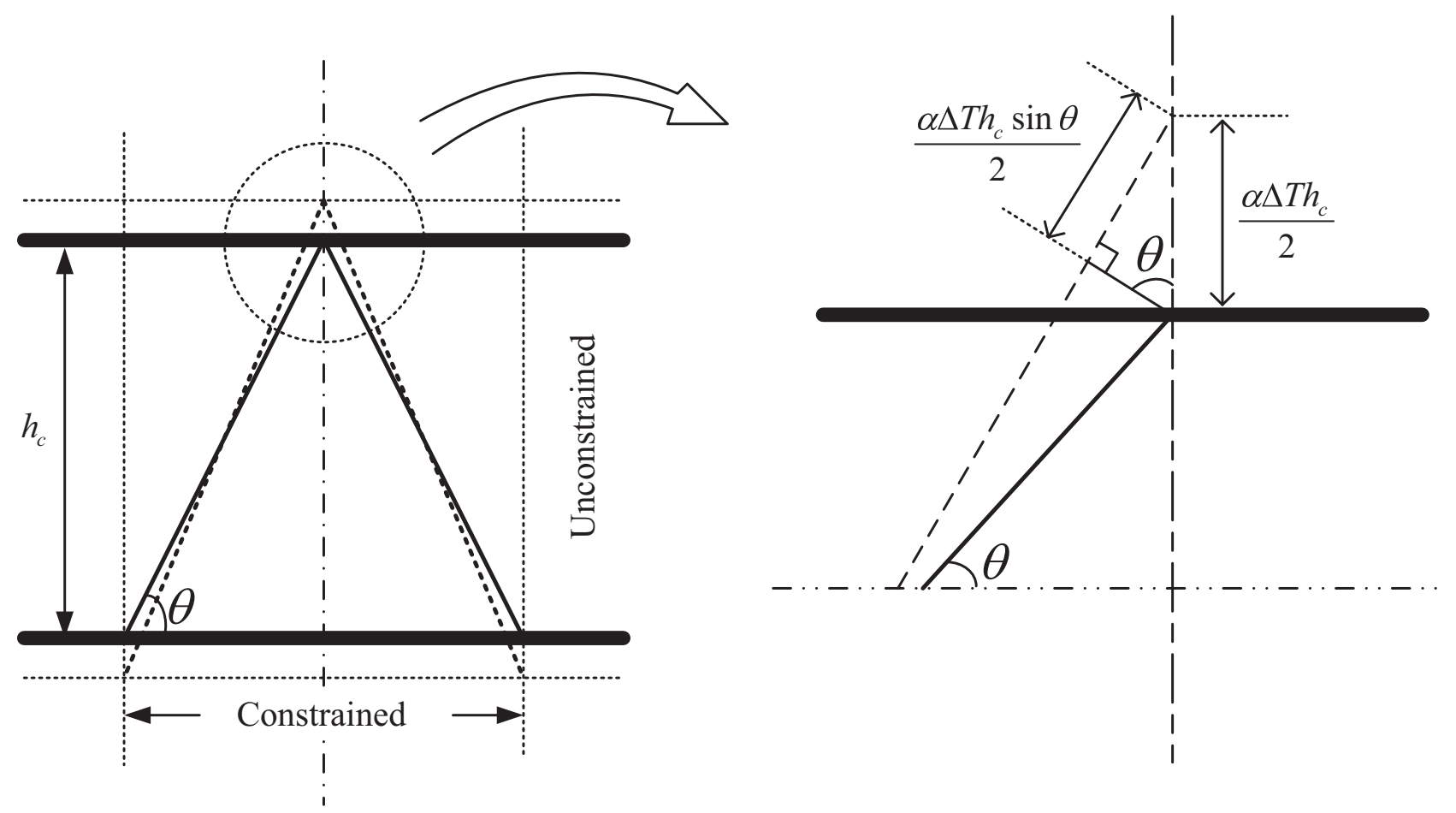

Fig. 4. Schematic of thermal mechanical deformations of the core member.

$$
\begin{aligned}
& \Delta T_{\text {tet }}^{C B}=\frac{\pi^{2} t_{c}^{2}}{24 h_{c}^{2} \alpha\left(1-\sin ^{2} \theta\right)} \\
& \Delta T_{\text {Kagome }}^{C B}=\frac{\pi^{2} t_{c}^{2}}{6 h_{c}^{2} \alpha\left(1-\sin ^{2} \theta\right)}
\end{aligned}
$$

\subsection{Core member yielding $(C Y)$}

For sandwich panels with stubby core members, CY will be the main failure mode instead of $\mathrm{CB}$. According to the deformation of the core member, the critical temperature rise that leads to $\mathrm{CY}$ is given by

$$
\Delta T=\frac{\epsilon_{y}}{\alpha\left(1-\sin ^{2} \theta\right)}
$$

It can be found from the comparison of Eq. (12) and Eq. (15) that the core member will never yield before the failure mode of FY. Therefore, this failure mode needs not to be considered in following sections.

\section{Failure mechanism maps of SPTC under thermal loading}

\subsection{SSSS boundary condition}

The preceding analysis on the failure behavior of SPTCs can be employed to generate failure mechanism maps. It is assumed that, in constructing such a map, the operative failure mode is one associated with the lowest critical temperature rise. When normalized by the edge length of the sandwich panel, dimensionless parameters can be expressed as

$\lambda_{\mathrm{c}}=t_{\mathrm{c}} / L$

$\lambda_{f}=t / L$

$$
\Lambda_{c}=h_{c} / L
$$

The corresponding weight index from Eq. (2) is

$\Psi=\frac{W}{\rho L}=2 \lambda_{f}+\eta \cdot \frac{\lambda_{c}^{2} \tan ^{2} \theta}{\Lambda_{c} \sin \theta}$

And the constraint based on the failure mode of SSSS SPTCs can be expressed as

$\frac{2 \alpha \Delta T}{(1-\mu) \pi^{2} \sin ^{3} \theta}\left(\frac{\left(1-\mu^{2}\right) \sin ^{3} \theta}{\left(\Lambda_{c}+\lambda_{f}\right)^{2}}+\frac{\beta \pi^{2} \Lambda_{c} \lambda_{f}}{\lambda_{c}^{2}}\right) \leq 1$

$\phi \cdot \frac{\alpha \Delta T(1+\mu) \Lambda_{c}^{2}}{\pi^{2} \lambda_{f}^{2}} \leq 1$

$\frac{\alpha \Delta T}{\epsilon_{y}} \leq 1 \quad(\mathrm{FY})$

$\varphi \cdot \frac{\alpha \Delta T \Lambda_{c}^{2}}{\pi^{2} \lambda_{c}^{2}}\left(1-\sin ^{2} \theta\right) \leq 1$

Dimensionless values of $\beta, \phi, \varphi$ and $\eta$ are governed by the topology of truss cores, which are summarized in Table 2.

Table 2

Parameters for constraint and objective functions in SSSS condition.

\begin{tabular}{lrrlll}
\hline Configurations & & Pyramidal & Tetrahedral & X-type & Kagome \\
\hline Global buckling & $\beta$ & 1 & $\sqrt{3}$ & 1 & $\sqrt{3}$ \\
Face buckling & $\phi$ & 12 & $54 / 7$ & $24 / 5$ & $14 / 27$ \\
Cores buckling & $\varphi$ & 24 & 24 & 6 & 6 \\
Weight function & $\eta$ & 2 & $2 \sqrt{3} / 3$ & 2 & $2 \sqrt{3} / 3$ \\
\hline
\end{tabular}




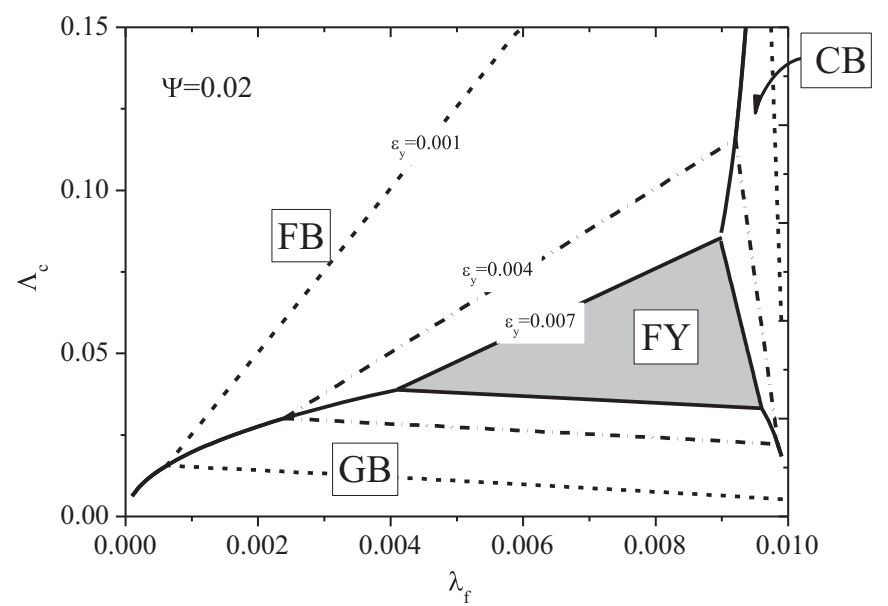

Fig. 5. Failure mechanism maps of pyramidal SPTCs under SSSS conditions with fixed weight index.

To generate failure mechanism maps, the core member thickness $\lambda_{\mathrm{c}}$ should be eliminated from constraint functions, making the maps rendering in coordinates face sheet thickness $\lambda_{\mathrm{f}}$ and core thickness $\Lambda_{c}$. When the weight index is fixed at 0.02 , the core member thickness can be written as

$\lambda_{c}^{2}=\frac{\left(0.02-2 \lambda_{f}\right) \Lambda_{c} \sin \theta}{\eta \tan ^{2} \theta}$

By sequentially equating pairs of constraint functions, domain
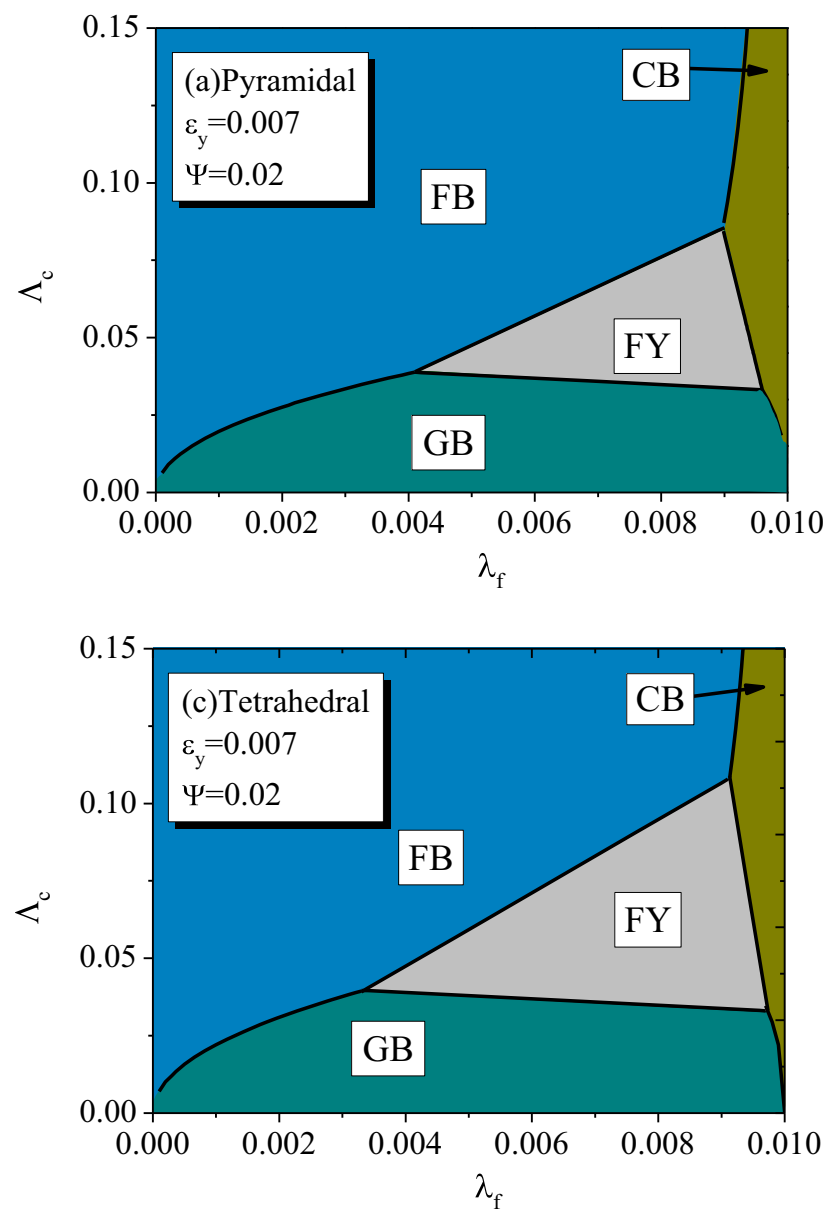

boundaries are obtained. With each domain, the load bearing capacity is calculated by Eqs. (18a-18d). In addition, failure maps of SSSS sandwich panel with pyramidal truss cores subjected to uniform thermal loading are shown in Fig. 5 when the yield strain $\varepsilon_{y}$ is $0.001,0.004$ and 0.007 respectively. It should be noted that the yield strain $\varepsilon_{y}$ significantly influences the location of boundaries between failure modes. The area of FY located in the center increases as the yield strain grows. Moreover, the increase of core thickness $\Lambda_{c}$ yields an improved resistance of global buckling, so the failure mode turns to FB from GB when the sandwich panel has small dimensionless face sheet thickness $\lambda_{f}$. When the face sheet thickness is large, CB will be the active failure mode for sandwich panel with higher core thickness.

Fig. 6 shows failure mechanism maps of SSSS sandwich panels with different truss core configurations when the yield strain is 0.007. It can be found that the failure mode of FB is more likely happened in sandwich panels with pyramidal and tetrahedral truss cores than those with X-type and Kagome configurations. This may due to additional connecting nodes between core members, which decrease the possibility of FY failure. And likewise, thermal stresses in pyramidal and tetrahedral truss cores more easily leads to $\mathrm{CB}$ failure, due to the longer core member (no intermediate nodes in the core member).

\subsection{CCCC boundary condition}

For the CCCC sandwich panel, only critical temperature of GB is different from those in SSSS conditions. As mentioned above, the critical load of GB cannot be solved by analytical expressions. Therefore, boundaries between the failure mode of GB and the
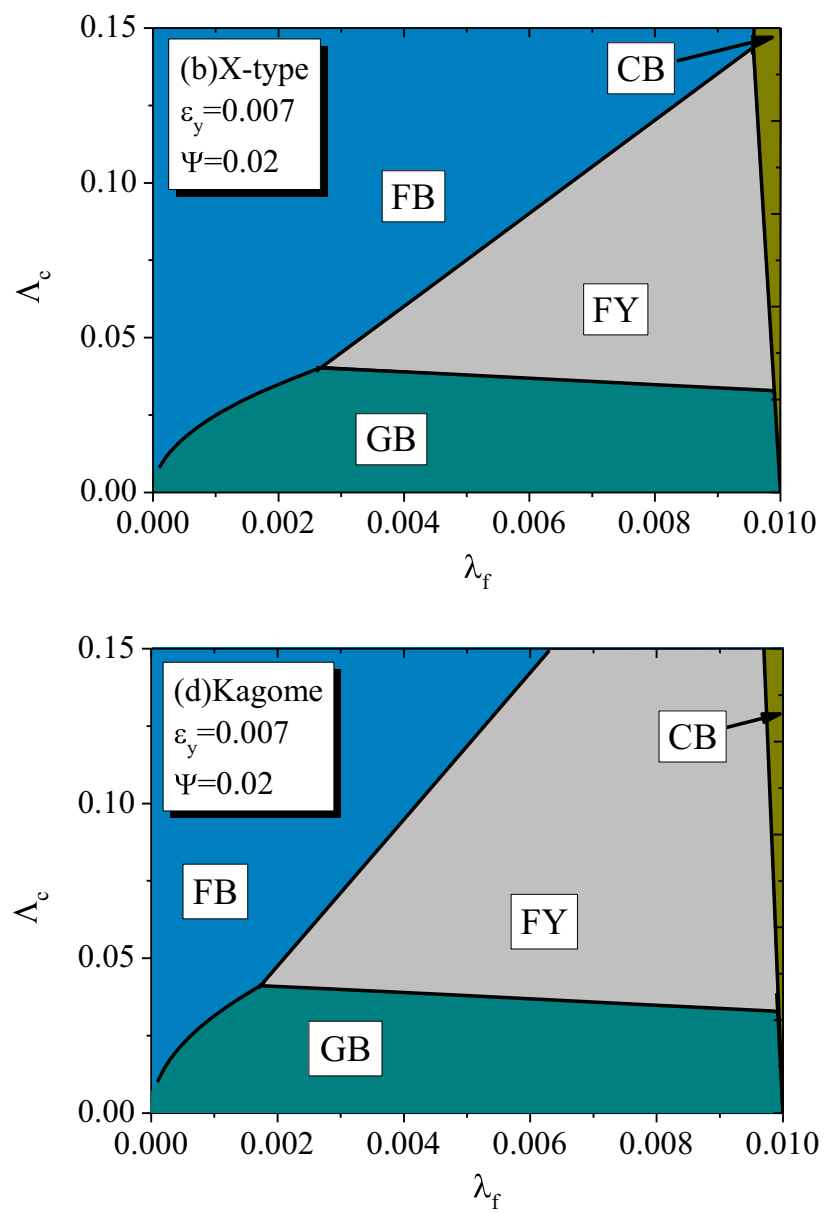

Fig. 6. Failure mechanism maps of different configuration SPTCs under SSSS conditions. 


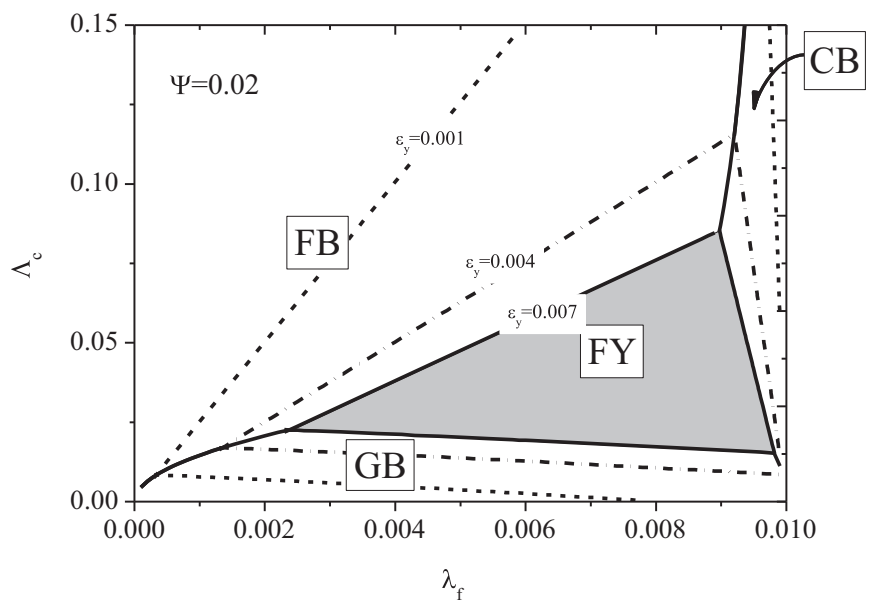

Fig. 7. Failure mechanism maps of pyramidal SPTCs under CCCC condition with fixed weight index.

other three failure modes described by Eqs. (18b-18d) are solved by a numerical program in Fortran code.

Fig. 7 shows failure maps of CCCC sandwich panels with pyramidal truss cores, when the yield strain $\varepsilon_{y}$ is $0.001,0.004$ and 0.007. Fig. 8 shows failure mechanism maps of CCCC sandwich panels with different truss core configurations when the yield strain is 0.007 . Under CCCC conditions, not only in-plane and outof-plane motions but also rotations at edges are restricted. Therefore the stability of SPTCs under CCCC condition is
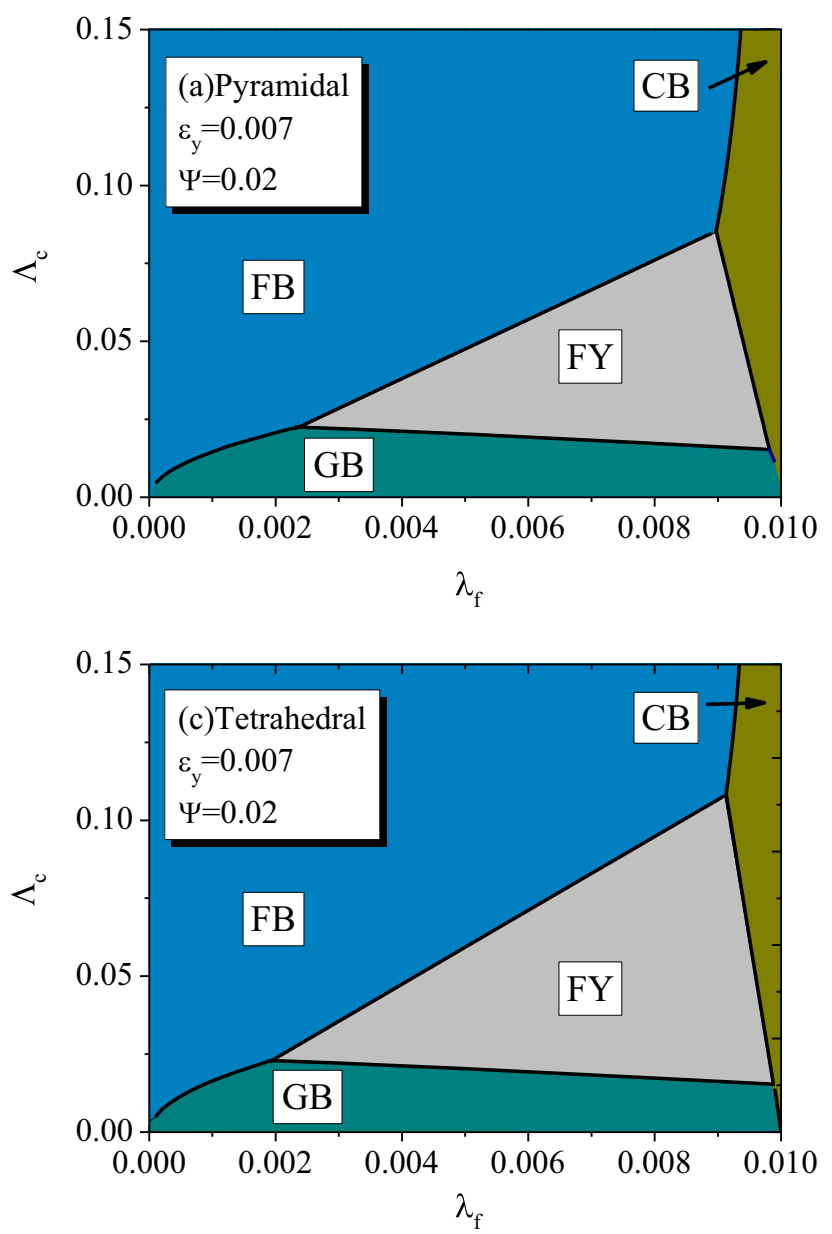

significantly improved [32]. The critical temperature of GB for the sandwich panel under CCCC condition is much higher than that under SSSS condition. As a result, global buckling is not easily happened in the sandwich panel with CCCC boundary condition.

\section{Optimal design of SPTCs}

\subsection{Optimization methods}

The analysis is extended to obtain the entire family of optimal designs for SPTCs subjected to uniform thermal loading. The goal is to find the minimum weight of SPTCs that can maintain structural integrity at a given thermal loading. However, it can be found from Eq. (18c) that the failure mode of FY is irrelevant to the geometrical configuration of SPTCs. It means when the temperature rise is lower than the critical temperature of FY, this constraint equation can be removed from the optimization model.

\subsubsection{Sequence linear programming (SLP) method}

It has been reported in some works that the optimal design of sandwich panel can be obtained by solving the confluence of various failure mechanisms $[22,23,34]$. This method may be appropriate for a linear problem. However, governing equations of different failure mechanisms (Eq. (18a-18d)) are nonlinear in nature. The global optimal result may not necessarily in the intersection of constraint equations for nonlinear programming problems and the numerical optimization method is imperative. Therefore, SLP method based on Lingo software is used in the
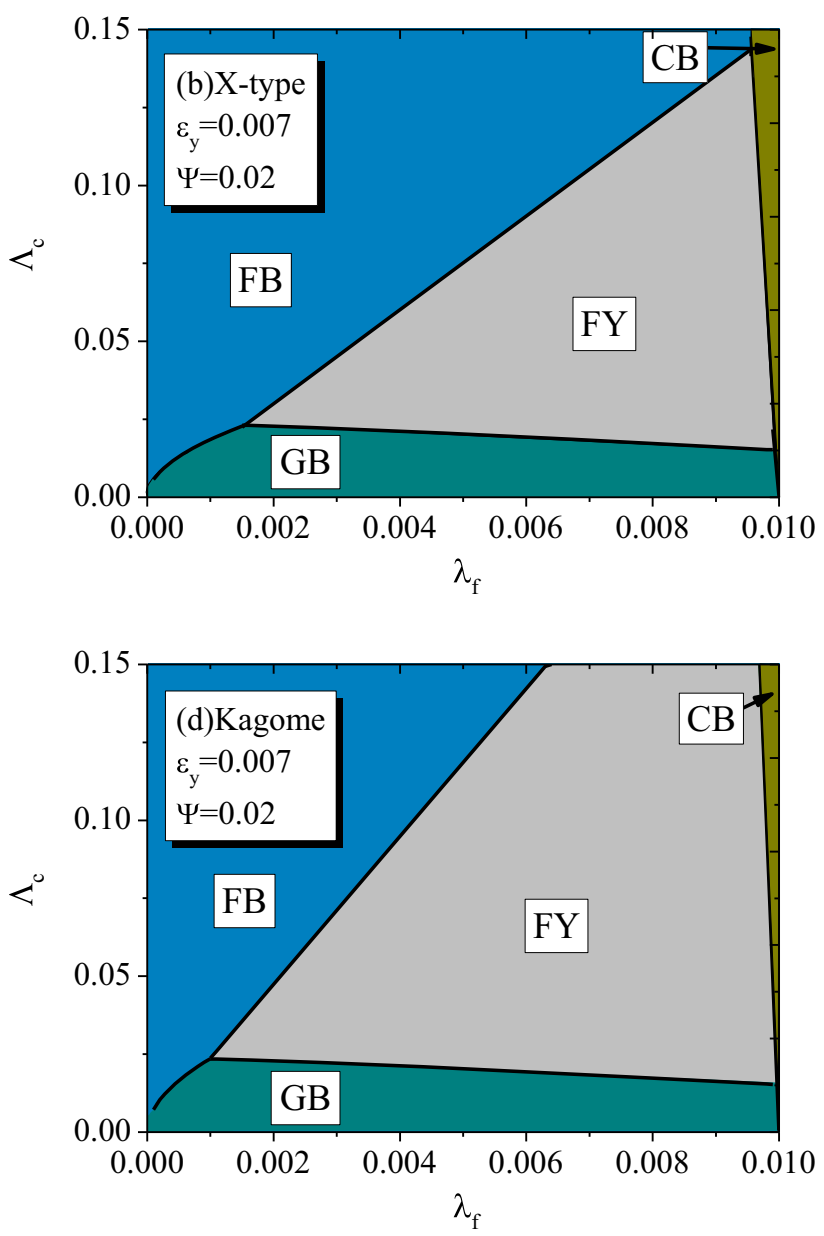

Fig. 8. Failure mechanism maps of different configuration SPTCs under CCCC condition. 
present paper to obtain an optimal design of SPTCs.

SLP is one of the most approximation optimization algorithms. By using Taylor expansions of the objective and constraint functions, the optimization is obtained through solving a series of approximate optimization problems. The general mathematic model of structure optimization is given by

Min $f(x)$

s.t. $h_{i}(x) \leq 0, \quad i=1, \ldots, M$

where $f(x)$ and $h_{i}(x)$ are objective functions and constraint functions respectively, $M$ and $x$ are the number of constraint functions and the design variable.

For the nonlinear optimization problem of Eq. (20), $x^{0}$ is assumed to be the initial estimated value. The objective function and constraint functions are expanded by Taylor series at the point of $x^{0}$.

$f(x) \approx f\left(x^{0}\right)+\nabla^{T} f\left(x^{0}\right)\left(x-x^{0}\right)=f^{0}(x)$

$h_{i}(x) \approx h_{i}\left(x^{0}\right)+\nabla^{T} h_{i}\left(x^{0}\right)\left(x-x^{0}\right)=h_{i}^{0}(x)$

Then, the linear optimization related to the nonlinear optimization problem of Eq. (20) is obtained

Min $f^{0}(x)$

s.t. $\quad h_{i}^{0}(x) \leq 0, \quad i=1, \ldots, M$

The optimal solution of the linear optimization problem $x^{1}$ can be obtained by solving Eq. (22). To obtained a better approximate solution, the functions of nonlinear optimization problem are expanded at the point of $x^{1}$, and another linear optimization problem is acquired. Finally, a series of approximate solutions $x^{k}$ can be obtained by repeating the process above. The iteration is assumed to be convergent when

$\left\|x^{k}-x^{k-1}\right\| \leq \varepsilon$

where $\varepsilon$ is the tolerance of sufficient small

\subsubsection{Comparison of optimization methods}

In order to compare different optimization methods, the confluence of the three failure modes of SSSS SPTCs are also solved by setting the three associated constraint equations Eqs. (18a-18b) and Eq. (18d) equal to unity. Fig. 9 shows optimal normalized

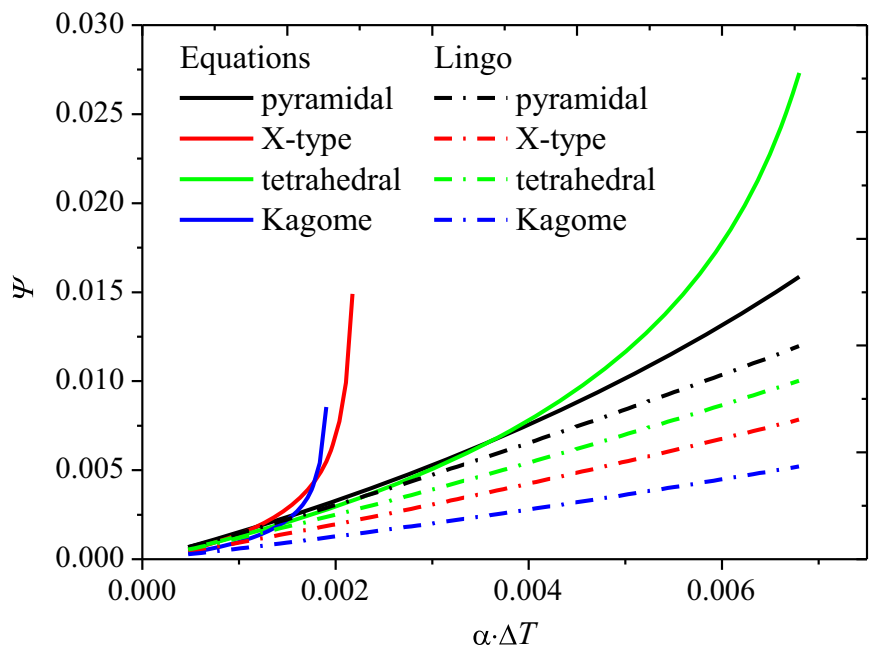

Fig. 9. Normalized weight per unit area for optimally designed SPTCs.

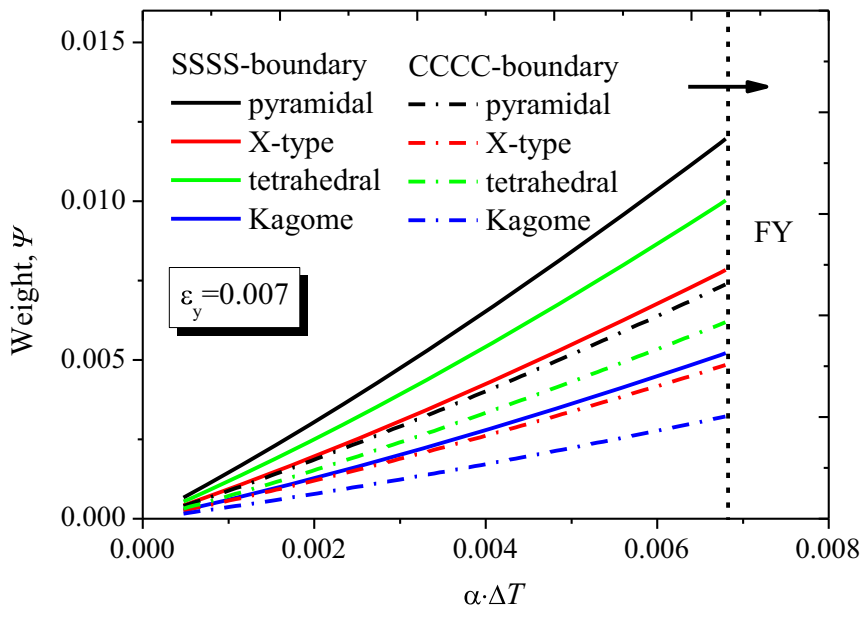

Fig. 10. Normalized weight per unit area for optimally designed SPTCs with SSSS and CCCC boundary conditions.

weights obtained by the two methods. It can be found that results from SLP are obviously lower than those from the confluence of the three failure modes, especially for the X-type and Kagome configuration. Therefore, the confluence of various failure modes is not the optimal design in the present case.

For CCCC SPTCs, the critical temperature of GB cannot be analytical solved. Therefore, the optimal design is obtained through the interaction between the software of Lingo and the numerical program in Fortran code. The model was created automatically by calling the file of Fortran Dynamic Link library, which was used to compute the critical temperature rise of CCCC SPTCs.

\subsection{Results and discussions}

Fig. 10 shows optimal designs of SSSS and CCCC sandwich panels with different configurations of truss cores from the numerical optimization model, when the yield strain is 0.007. As mentioned above, the resistance to GB of a CCCC SPTC is higher than that in SSSS condition [32]. Besides, critical bucklling loads of SPTCs under other four failure mechanisms are irrelevant to the constraint of rotations at the boundaries. Therefore, the weight per unit area of the CCCC sandwich panel is smaller. Due to the effect of intermediate nodes in the core member, sandwich panels with Kagome and X-type truss cores have higher strength to $\mathrm{CB}$. Therefore, the normalized weight of sandwich panels with Kagome and $\mathrm{X}$-type truss cores are dramatically lower than those with tetrahedral and pyramidal truss cores. Some similar conclusions also can be found on the behavior of SPTCs under compressive and bending loadings $[15,16,35]$. In addition, the distribution of nodes between cores and the face sheet influences the critical load of FB and then affect the optimal weight of the SPTC. The performance of configurations in the order of decreased minimum weight is: pyramidal, tetrahedral, X-type and Kagome. Fig. 11 shows the normalized geometrical parameters for optimally designed SPTCs with SSSS and CCCC conditions. It can be found that, as shown in Fig. 11a, the variations of optimal normalized face sheet thickness $\lambda_{f}$ are nearly linear to the uniform thermal loading $\Pi_{t}$. And, $\lambda_{f}$ of SSSS SPTCs is obviously larger than those in CCCC condition. Similar conclusions can be obtained in the analysis of the core member thickness, as Fig. 11b shows. However the normalized core thickness $\Lambda_{c}$, which has a nonlinear relationship with the thermal loading $\Pi_{t}$, is an exception. As the thermal loading increases, the growth rate for the core thickness is decreasing.

Moreover, the contribution of the truss core is independent of 

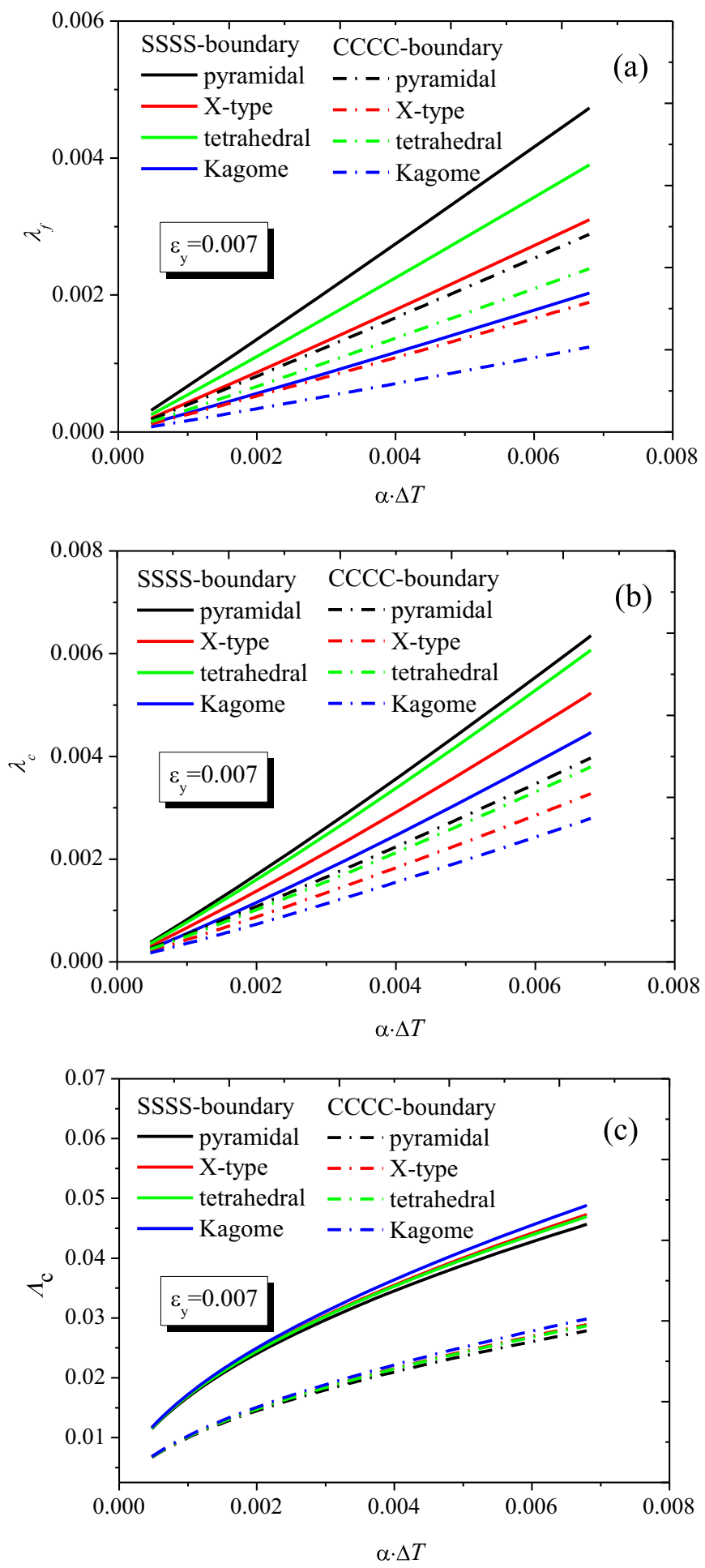

Fig. 11. Normalized geometrical parameters for optimally designed SPTCs with SSSS and CCCC boundary conditions. (a) face sheet thickness, (b) core member thickness, (c) core thickness.

the face sheet, and it is given by

$\Psi_{\text {cores }}=\eta \cdot \frac{\lambda_{c}^{2} \tan ^{2} \theta}{\Lambda_{c} \sin \theta}$

The result of optimal normalized core weight versus the thermal loading is plotted in Fig. 12a. The weight of truss cores has a nonlinear relationship with the thermal loading. In contrast to the
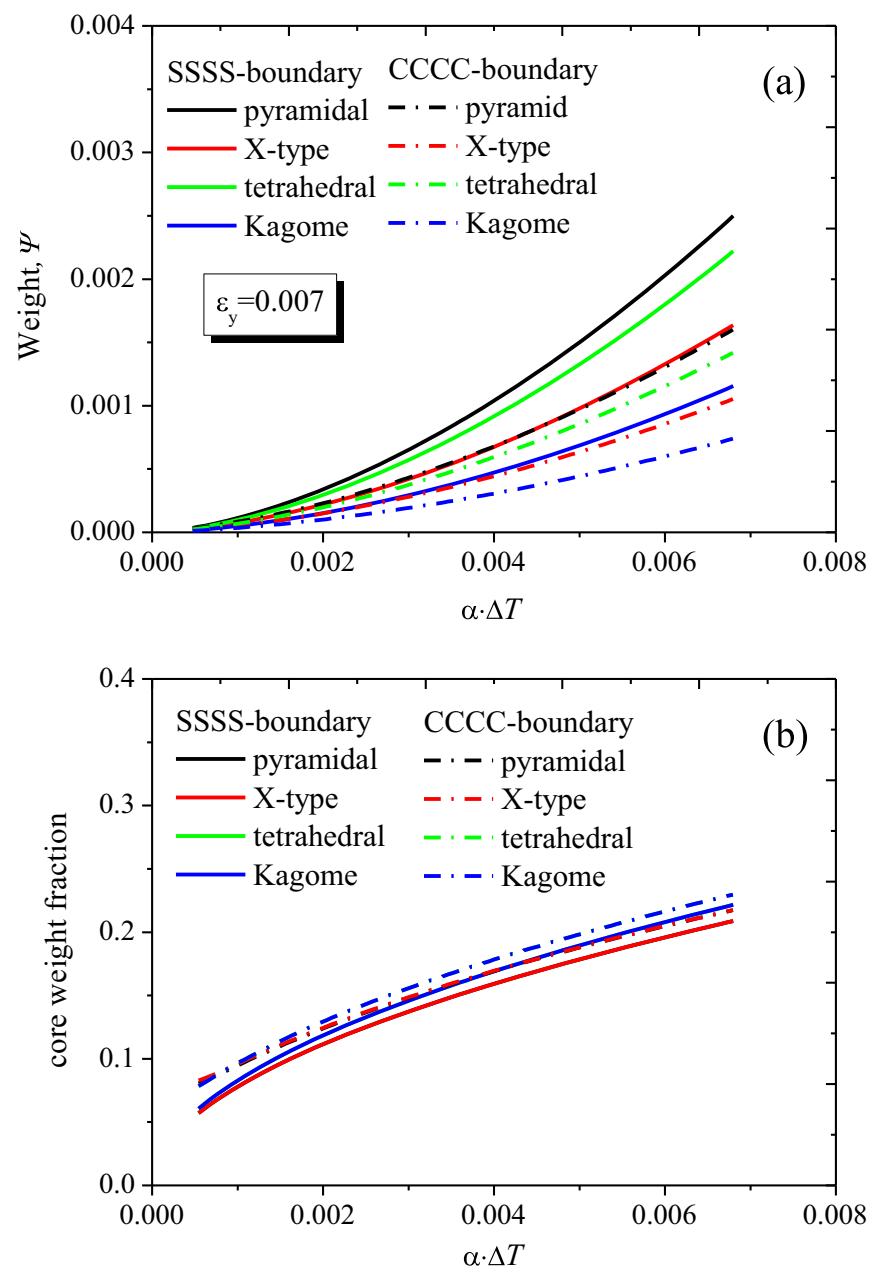

Fig. 12. Truss core contributions to the weight of the sandwich panel. (a) normalized core weight versus thermal loading (b)Core weight fraction (relative to total weight) versus thermal loading.

core thickness, the core weight grows more rapidly with the increase of the thermal loading. Therefore, as Fig. 12b shows, the truss core weight fraction increases when the thermal loading grows. It means that the sandwich panel should have a higher fraction of truss core weight relative to the total panel weight when subjected to a more severe thermal loading.

Fig. 13a shows the ratio of normalized core member thickness of the optimal design to that from the critical buckling temperature rise from Eqs. (14a-14d). It can be found that, to obtain the optimal design of SPTCs subjected to uniform thermal loading, the core member thickness should greater than the critical thickness. However, as shown in Fig. 13b, it should be critical value for the core thickness and the face sheet thickness.

Deterioration of mechanical properties of the metallic material, in particular the yield stress and elastic modulus, is an obvious effect of high temperature on a structure. The coefficient of thermal expansion $\alpha$ is less affected by the temperature and will not be considered. It can be found from Eqs. (18a-18d) that critical loads are irrelevant to variations of mechanical properties with temperatures, except for the failure mode of FY. When considering the variation of mechanical properties, the constraint equation of FY become

$\frac{\alpha \Delta T}{\epsilon_{y}\left(T_{0}+\Delta T\right)} \leq 1$ 

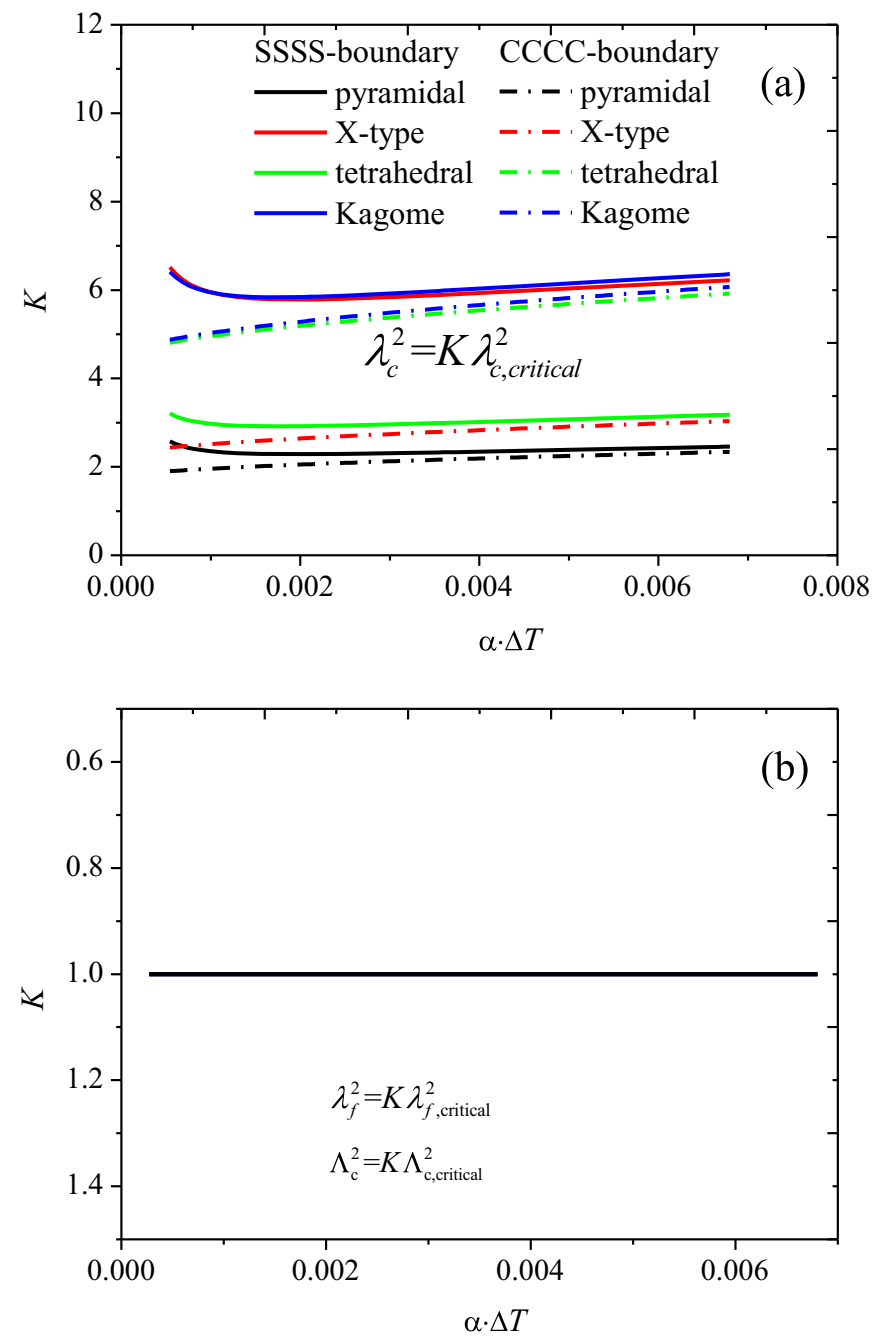

Fig. 13. Ratios of normalized geometric parameters for optimally designed SPTCs to critical values. (a) core member thickness, (b) core thickness and face sheet thickness.

Where $T_{0}$ is the room temperature. Taken AISI304 stainless steel as an example, the yield strain versus temperatures can be written in a polynomial function

$\varepsilon_{y}=P_{0}+P_{1} T+P_{2} T^{2}+P_{3} T^{3}+P_{4} T^{4}$

where $P_{0}, P_{1}, P_{2}, P_{3}$, and $P_{4}$ are constant coefficients of the polynomial function. The critical load of the FY can be obtained by solving Eq. (25) and Eq. (26). Then, failure mechanism maps and the optimal design of SPTC made of AISI304 stainless steel can be obtained according to this critical load. This method is applicable to SPTCs made of other metallic materials.

\section{Conclusions}

The response of SPTCs subjected to uniform thermal loading has been studied analytically and numerically. Analytical formulae are developed for the failure strength of the SPTC and five possible failure modes for the four different configurations of truss cores are identified. Failure mechanism maps for the SPTC made from a single metallic material are developed when the dimensionless weight index are fixed. Using these failure modes as constraint conditions, sandwich panels with different configuration truss cores are optimally designed by the numerical optimization model based on Lingo. Due to the high strength to the GB, SPTCs with CCCC boundary conditions are more efficient than those with SSSS boundary conditions. The performance of sandwich panels with Kagome and X-type truss cores are superior to the other two configurations. In addition, sandwich panels should have a higher fraction of truss cores when subjected to a more severely thermal loading. The core member thickness should be larger than the critical value to reduce the weight of the SPTC.

\section{Acknowledgments}

Financial supports from National Natural Science Foundation of China (Grant nos. 91016025, 11472276, and 11332011) are gratefully acknowledged.

\section{References}

[1] Ashby M, Brechet Y. Designing hybrid materials. Acta Mater 2003;51:5801-21.

[2] Cui X, Zhao L, Wang Z, Zhao H, Fang D. Dynamic response of metallic lattice sandwich structures to impulsive loading. Int J Impact Eng 2012;43:1-5.

[3] Deshpande V, Fleck N, Ashby M. Effective properties of the octet-truss lattice material. J Mech Phys Solids 2001;49:1747-69.

[4] Lu T, Valdevit L, Evans A. Active cooling by metallic sandwich structures with periodic cores. Prog Mater Sci 2005·50:789-815.

[5] Wadley HNG. Multifunctional periodic cellular metals. Philos Trans R Soc A 2006;364:31-68.

[6] Biagi R, Bart-Smith H. Imperfection sensitivity of pyramidal core sandwich structures. Int J Solids Struct 2007;44:4690-706.

[7] Cote F, Biagi R, Bart-Smith H, Deshpande VS. Structural response of pyramidal core sandwich columns. Int J Solids Struct 2007;44:3533-56.

[8] Chiras S, Mumm DR, Evans AG, Wicks N, Hutchinson JW, Dharmasena K, et al. The structural performance of near-optimized truss core panels. Int J Solids Struct 2002;39:4093-115.

[9] Deshpande VS, Fleck NA. Collapse of truss core sandwich beams in 3-point bending. Int J Solids Struct 2001;38:6275-305.

[10] Kooistra GW, Queheillalt DT, Wadley HNG. Shear behavior of aluminum lattice truss sandwich panel structures. Mat Sci Eng a-Struct 2008;472:242-50.

[11] Wallach JC, Gibson LJ. Mechanical behavior of a three-dimensional truss material. Int J Solids Struct 2001;38:7181-96.

[12] Lee BK, Kang KJ. A parametric study on compressive characteristics of wire-woven bulk Kagome truss cores. Compos Struct 2010;92:445-53.

[13] Lim JH, Kang KJ. Mechanical behavior of sandwich panels with tetrahedral and Kagome truss cores fabricated from wires. Int J Solids Struct 2006:43:5228-46.

[14] Yan HB, Zhang QC, Lu TJ, Kim T. A lightweight X-type metallic lattice in single-phase forced convection. Int J Heat Mass Transf 2015;83:273-83.

[15] Zhang QC, Chen AP, Chen CQ Lu TJ. Ultralight X-type lattice sandwich structure (II): micromechanics modeling and finite element analysis. Sci China Ser E 2009;52:2670-80.

[16] Zhang QC, Han YJ, Chen CQ, Lu TJ. Ultralight X-type lattice sandwich structure (I): concept, fabrication and experimental characterization. Sci China Ser E $2009 \cdot 52 \cdot 2147-54$

[17] Zhang OC, Lu TJ. Experimental and simulated compressive properties of work-hardened X-type lattice truss structures. Acta Mech Solida Sin 2012;25:111-6.

[18] Deshpande VS, Ashby MF, Fleck NA. Foam topology bending versus stretching dominated architectures. Acta Mater 2001;49:1035-40.

[19] Rathbun HJ, Wei Z, He MY, Zok FW, Evans AG, Sypeck DJ, et al. Measurement and simulation of the performance of a lightweight metallic sandwich structure with a tetrahedral truss core. J Appl Mechanics-Trans ASME 2004;71:368-74.

[20] Li M, Wu LZ, Ma L, Wang B, Guan ZX. Structural response of all-composite pyramida truss core sandwich columns in end compression. Compos Struct 2011:93:1964-72.

[21] Wicks N, Hutchinson JW. Optimal truss plates. Int J Solids Struct 2001:38:5165-83.

[22] Zok FW, Waltner SA, Wei Z, Rathbun HJ, McMeeking RM, Evans AG. A protocol for characterizing the structural performance of metallic sandwich panels: application to pyramidal truss cores. Int J Solids Struct 2004;41:6249-71.

[23] Rathbun HJ, Zok FW, Evans AG. Strength optimization of metallic sandwich panels subject to bending. Int J Solids Struct 2005;42:6643-61.

[24] Singer J, Arbocz J, Weller T. Buckling experiments: experimental methods in buckling of thin-walled structures. shells, built-up structures, composites and additional topics. John Wiley \& Sons, Hoboken, NJ; 2002.

[25] Mossavarali A, Eslami M. Thermoelastic buckling of plates with imperfections based on a higher order displacement field. J Therm Stress 2002;25:745-71.

[26] Ovesy H, Taghizadeh M, Kharazi M. Post-buckling analysis of composite plates containing embedded delaminations with arbitrary shape by using higher order shear deformation theory. Compos Struct 2012;94:1243-9.

[27] Reissner E. On small bending and stretching of sandwich-type shells. Int J Solids Struct 1977; $13: 1293-300$.

[28] Shariyat M. Thermal buckling analysis of rectangular composite plates with temperature-dependent properties based on a layerwise theory. Thin-Walled Struct 2007;45:439-52.

[29] Shen H-S. Thermal postbuckling behavior of shear deformable FGM plates with 
temperature-dependent properties. Int J Mech Sci 2007;49:466-78.

[30] Shiau L-C, Kuo S-Y. Thermal buckling of composite sandwich plates. Mech Based Des Struct Mach 2004:32:57-72.

[31] Rakow JF, Waas AM. Thermal buckling of metal foam sandwich panels for convective thermal protection systems. J Spacecr Rocket 2005;42:832-44.

[32] Yuan W, Wang X, Song H, Huang C. A theoretical analysis on the thermal buckling behavior of fully clamped sandwich panels with truss cores. J Therm Stress
2014;37:1433-48.

[33] Yuan W, Song H, Wang X, Huang C. Experimental investigation on thermal buckling behavior of truss-core sandwich panels. AIAA J 2014:1-10.

[34] Zok FW, Rathbun HJ, Wei Z, Evans AG. Design of metallic textile core sandwich panels. Int J Solids Struct 2003;40:5707-22.

[35] Hyun S, Karlsson AM, Torquato S, Evans AG. Simulated properties of Kagome and tetragonal truss core panels. Int J Solids Struct 2003;40:6989-98. 